\title{
Fuzzy Synthesis Judgment Evaluating Model of Project Practice Teaching
}

\author{
Hua Cao \\ Jiangxi Institute of Fashion Technology \\ Nanchang, China \\ 406563256@qq.com
}

\author{
Hai Yin \\ Jiangxi Institute of Fashion Technology \\ Nanchang, China \\ 3056453@qq.com
}

\begin{abstract}
Project practice teaching assessment investigate comprehensively that students master knowledge application and new knowledge, however, traditional test paper can't meet requirement. This paper puts forward fuzzy synthesis evaluation model, which assesses roundly student's performance in project practice from students factor, advisor and expert point of view, then it makes a reasonable and fair evaluation for students' performance. The practice shows that the model can assess reasonably student's grade in project practice, and it's recognized by students and teachers.
\end{abstract}

Keywords- Software project practice; Fuzzy synthesis judgment; performance evaluation

\section{INTRODUCTION}

Project practice is an important phase in the university teaching process, the aim is to cultivate students ability of handed operation and analysis as well as creativity, to improve their software comprehensive ability, help student to find a good job after graduation[1-2]. Junior and senior student are asked to join the project, A team consisting of 4-5 student should finish a project within fixed time according strictly software engineer idea with project guiding teaching method (the project can be given project by teacher, creative project and supposed project), at last student should finish a standard file. Every member in the team has clear task, the project is typical, objective and inspired[2-4]. In the completion process of project, Students should make full use of knowledge and internet resource to solve different problems, meanwhile, They should have spirit of team cooperation. Student's non-knowledgeable factor and comprehensive ability will developed well, such as creative application ability, communication skill as well as cooperation conscious

Software project practice is a course, which will be given reasonable score after test, However, the traditional test method is teacher give score according students' daily work. Written test score as well as experience score. Despite the test method is relatively reasonable, in the process of project practice, students will use broader knowledge, the traditional method can't meet the need. In addition, teacher is difficult to evaluate student in the term of students' different ability. The text put forward a evaluate method of project practice based on the fuzzy synthesis judgment[5-6]. The method divide evaluating indicators into different angels, each factor should be assessed, The result of evaluation considering as upper class will be assessed again, the method avoid the disadvantage of the one fuzzy evaluation method. According to students' performance in the project practice, using the multi-level fuzzy comprehensive evaluation method to evaluate comprehensively student's achievement can make it more rational and just, Meanwhile, It will promote students' study enthusiasm obviously. So it has more practice value. In the paper, the secondary fuzzy comprehensively appraisal mode of project practice teaching appraisal method has been put forward, we determine the indicators factor, according to our college actual situation, describe in detail the students' achievement assessment in the project practice teaching process with two fuzzy comprehensive appraisal method.

\section{THE MODEL OF SECONDARY FUZZY COMPREHENSIVE EVALUATION}

The model of secondary fuzzy comprehensive evaluation includes six steps: appointing index set, assigning index weight, appointing remark set, implementing first-degree fuzzy comprehensive evaluation and secondary fuzzy comprehensive evaluation, assessing the final result. The following are detailed description.

\section{A. Appointing index set}

The factors of evaluating students' achievement have a lot of aspects. The choices of evaluating index are based on plentiful teaching experience and widely absorbing the teaching fruit of experts, decided by conclusion and analysis. Thus, to classify the main factors affected evaluation, which is to set the index set as like formula (1).

$$
A=\left\{A_{1}, A_{2}, \ldots, A_{s}\right\}
$$

In which, $A$ is the object of evaluation, and the index are divided as $s$ subsets which satisfy the formula (2).

$$
\bigcup_{i=1}^{s} A_{i}=A, \quad A_{i} \cap A_{j}=\phi,(i \neq j)
$$

Each index subset $A_{i}(i=1,2, \cdots, s)$ has $n_{i}$ sub index which satisfies the requirements of formula (3) and (4).

$$
A_{i}=\left\{A_{i 1}, A_{i 2}, \ldots, A_{i n_{i}}\right\},(i=1,2, \ldots s)
$$




$$
\sum_{i=1}^{s} n_{i}=n
$$

$A_{i j}\left(i=1,2, \cdots, s, j=1,2, \cdots, n_{i}\right)$ is the sub index which will affect object $X$, the number of index is $n$.

\section{B. Assigning index weight}

To assign a weight to every index and sub index to correspond to index set and index subset as formula (5) and (6) shown, and the sum of all weight is 1, as formula (7) shown.

$$
\begin{gathered}
A=\left\{a_{1}, a_{2}, \ldots, a_{s}\right\} \\
A_{i}=\left\{a_{i 1}, a_{i 2}, \ldots, a_{i n_{i}}\right\}, \quad(i=1,2, \ldots s) \\
\sum_{i=1}^{s} a_{i}=1, \quad a_{i}=\sum_{j=1}^{n_{i}} a_{i n_{i}}
\end{gathered}
$$

\section{Appointing remark set}

Remark set is the set composed by elements of every possible results of total evaluation, generally shown as $V=\left(v_{1}, v_{2}, \cdots, v_{m}\right)$, where in $v_{i}(j=1,2, \cdots, m)$ is the remark level, represented by excellent, good, middling, pass, failure, you can use values (percentile system ) in the form of express.

\section{Implementing first-degree fuzzy comprehensive evaluation}

For every subset $A_{i}$, comprehensively evaluating based on the first-degree fuzzy comprehensive evaluation, includes the following two steps.

(a) Building the relation matrix $\mathrm{R}$ of evaluating index and remark, shown as formula (8).

$$
R_{i}=\left[\begin{array}{cccc}
r_{i 11} & r_{i 12} & \ldots & r_{i 1 m} \\
r_{i 21} & r i_{22} & \ldots & r_{i 2 m} \\
r_{i 31} & r i_{32} & \ldots & r_{i 3 m} \\
\ldots & \ldots & \ldots & \ldots \\
r_{i n 1} & r i_{n 2} & \ldots & r_{i n m}
\end{array}\right]
$$

The relation matrix can be built based on the facts, or on the statistics of the facts.

(b) Computing the result of the $i$ th index, shown as formula (9).

$$
B_{i}=A_{i} \circ R_{i}=\left(a_{i 1}, a_{i 2}, \ldots, a_{i n_{i}}\right) \circ\left[\begin{array}{cccc}
r_{i 11} & r_{i 12} & \ldots & r_{i 1 m} \\
r_{i 21} & r i_{22} & \ldots & r_{i 2 m} \\
r_{i 31} & r i_{32} & \ldots & r_{i 3 m} \\
\ldots & \ldots & \ldots & \ldots \\
r_{i n 1} & r i_{n 2} & \ldots & r_{i n m}
\end{array}\right]=\left(b_{i 1}, b_{i 2}, \ldots, b_{i m}\right)
$$

The mark " 。" is the fuzzy operator symbol, using the common operation method, thus is the computation of the fuzzy matrix corresponds to the multiplication of the common matrix, however, the sum is the max, and the product is the $\min$.

\section{E. Implementing secondary fuzzy comprehensive evaluation}

Using the fuzzy evaluation results of every subset into the secondary fuzzy comprehensive evaluation, the firstdegree fuzzy comprehensive evaluation matrix is shown as formula (10). Based on the result of the first-degree fuzzy comprehensive evaluation, the secondary fuzzy comprehensive evaluation value $B$, is shown as formula (11).

$$
\begin{gathered}
R=\left[\begin{array}{c}
B_{1} \\
B_{2} \\
\ldots \\
B_{s}
\end{array}\right]=\left[\begin{array}{c}
A_{1} \circ R_{1} \\
A_{2} \circ R_{2} \\
\ldots \\
A_{s} \circ R_{s}
\end{array}\right] \\
B=A \circ R=A \circ\left[\begin{array}{c}
A_{1} \circ R_{1} \\
A_{2} \circ R_{2} \\
\ldots \\
A_{s} \circ R_{s}
\end{array}\right]=\left(b_{1}, b_{2}, \ldots, b_{m}\right)
\end{gathered}
$$

\section{$F$. Assessing the final result}

$B$ is the all-around value of all index, Through the normalization of $b_{k}(k=1,2, \cdots, m)$, we get $B^{\prime}$ as shown in formula (12).

$$
b_{k}^{\prime}=\frac{b_{k}}{\sum_{k=1}^{m} b_{k}}
$$

After the normalization, computing the evaluation results, shown in formula (13), we get the value of $w$ and the remark level.

$$
w=b_{k}^{\prime} \times v^{T} \text {. }
$$


III. PROJECT PRACTICE TEACHING ASSESSMENT OF THE SECONDARY FUZZY COMPREHENSIVE EVALUATION MODEL

Project practice teaching see about students 'knowledge, a lot of factors that can not be quantitatively described, Here using two comprehensive evaluation model for students to judge the grade, students receive a comprehensive score, following their elaborate.

\section{A. Measuring the software engineering practice project evaluation index}

In the view of teaching management, the assessment of student performance including the performance of the normal study, project design and development process, the quality of completed projects, etc., so there are a lot of evaluation, for the convenience, we will select 3 indicators from the student self-evaluation, select five indicators from teacher evaluation. the four indicators from experts .which constitute a select set of indicators, namely, A1 = (student self-evaluation indicators subset, teacher subset evaluation, expert evaluation index subset), students self-evaluation indicators subset $\mathrm{A} 1=$ (Study of the initiative and enthusiasm, spirit of team cooperation, individual contributions), teacher evaluation subset $\mathrm{A} 2=$ (attendance, attitude toward study, knowledge of project development technology, independent thinking and problem-solving skills, project completion time), the expert evaluation index subset A3 = (rationality of project design, quality of project completed, normative documents of project, the application value).

\section{B. Assigning of weight and relation of evaluation}

Index weight always adopt expert advice or Delphi method, according to experts tick at the consultation table, then the average and standard deviation of indicators can be calculated after statistical process. use of expert advisory method, combined with teaching experience, to calculate the index weight, get personal, teacher, expert weights were: A $=(0.2,0.4,0.4)$, the corresponding subset of the various indicators : $\mathrm{A} 1=\{0.04,0.08,0.08\}, \mathrm{A} 2=\{0.04,0.08$, $0.12,0.08,0.08\}, \mathrm{A} 3=\{0.08,0.12,0.12,0.08\}$. our college adopt 5 points system in the evaluation of practice teaching process. these are excellent, good, medium, pass, fail, or use percentage system, but before credited to the report card, the evaluation should be converted into percentile according to the standard system (conversion standard may be different in different university), excellent, good, medium, pass, fail are corresponding to $95,85,75,65,55$ points, Thus evaluation collection $\mathrm{V}=$ (excellent, good, medium, pass, fail), corresponding values were: $95,85,75,65,55$.In accordance with project completion in the whole process of recording, corresponding of student self-evaluation indicators and reviews is R1, corresponding relations of teacher evaluation and reviews is R2, corresponding relations of expert evaluation indicators and reviews is R3, respectively as follows:

$$
\begin{gathered}
R_{1}=\left[\begin{array}{ccccc}
0.6 & 0.3 & 0.1 & 0 & 0 \\
0.8 & 0.2 & 0 & 0 & 0 . \\
0.7 & 0.2 & 0.1 & 0 & 0
\end{array}\right] R_{2}=\left[\begin{array}{ccccc}
0.8 & 0.2 & 0 & 0 & 0 \\
0.8 & 0.2 & 0 & 0 & 0 \\
0.6 & 0.3 & 0.1 & 0 & 0 . \\
0.6 & 0.2 & 0.1 & 0.1 & 0 \\
0.7 & 0.1 & 0.1 & 0.1 & 0
\end{array}\right] \\
R_{3}=\left[\begin{array}{lllll}
0.8 & 0.2 & 0 & 0 & 0 \\
0.7 & 0.3 & 0 & 0 & 0 \\
0.6 & 0.2 & 0.1 & 0.1 & 0 \\
0.5 & 0.3 & 0.1 & 0.1 & 0
\end{array}\right]
\end{gathered}
$$

\section{Judgment of result}

Based on the secondary fuzzy comprehensive evaluation model, from formula (9), we get the results of all evaluation index and the results are $B_{1}, B_{2}$ and $B_{3}$.

$$
\begin{gathered}
B_{1}=A_{1}{ }^{\circ} R_{1}=(0.04,0.08,0.08)^{\circ}\left[\begin{array}{ccccc}
0.6 & 0.3 & 0.1 & 0 & 0 \\
0.8 & 0.2 & 0 & 0 & 0 \\
0.7 & 0.2 & 0.1 & 0 & 0
\end{array}\right]=(0.08,0.08,0.08,0,0) \\
B_{2}=A_{2}{ }^{\circ} R_{2}=(0.4,0.08,012,0.08,0.08)\left(\begin{array}{lllll}
0.8 & 02 & 0 & 0 & 0 \\
0.8 & 02 & 0 & 0 & 0 \\
06 & 03 & 01 & 0 & 0 \\
06 & 02 & 01 & 01 & 0 \\
07 & 01 & 01 & 01 & 0
\end{array}\right]=(012,012,01,008,0) \\
\left.B_{3}=A_{3}{ }^{\circ} R_{3}=(0.08,0.12,0.12,0.08)\right)^{0}\left[\begin{array}{lllll}
0.8 & 0.2 & 0 & 0 & 0 \\
0.7 & 0.3 & 0 & 0 & 0 \\
0.6 & 0.2 & 0.1 & 0.1 & 0 \\
0.5 & 0.3 & 0.1 & 0.1 & 0
\end{array}\right]=(0.12,0.12,0.1,0.1,0)
\end{gathered}
$$

Based on the values of $B_{1}, B_{2}, B_{3}$ and formula (10), we get relation matrix $R$. Then based on $A$ and $R$, using formula (11), we get the value of $B$.

$$
R=\left[\begin{array}{ccccc}
0.08 & 0.08 & 0.08 & 0 & 0 \\
0.12 & 0.12 & 0.1 & 0.08 & 0 \\
0.12 & 0.12 & 0.1 & 0.1 & 0
\end{array}\right]
$$

$$
B=A \circ R=(0.2,0.4,0.4)^{\circ}\left[\begin{array}{ccccc}
0.08 & 0.08 & 0.08 & 0 & 0 \\
0.12 & 0.12 & 0.1 & 0.08 & 0 \\
0.12 & 0.12 & 0.1 & 0.1 & 0
\end{array}\right]=(0.12,0.12,0.1,0.1,0)
$$


Based on $B$, through normalization, using formula (12), we get $B^{\prime}=(0.273,0.273,0.227,0.227,0)$, then using formula (13) to find the value of $w$.

$$
w=(0.273,0.273,0.227,0.227,0) \times\left(\begin{array}{c}
95 \\
85 \\
75 \\
65 \\
55
\end{array}\right)=80.92
$$

Based on the value of $w$, the mark of this student' score is 80.92 , Therefore, we can conclude that this model can be applied in the performance evaluation of software projects practice teaching, similarly, this model is also applicable to performance evaluation of other items' project practice teaching.

\section{CONCLUSION}

The performance evaluation in project practice teaching aspect requires a comprehensive and integrated consideration of various influencing factors, but also need to distinguish between primary and secondary relations, so that we can make a more reasonable scientific assessment of student achievement,. Teaching practice has proved that students' performance is assessed based on two fuzzy comprehensive evaluation can be more objective, comprehensive and fair, and this approach put much descriptive qualitative assessment changed to quantified and formality scientific evaluation, makes the evaluation more reliable, The method overcome the disadvantage of subjective randomness and one-sidedness of traditional approach , at the same time can effectively enhance students normally study enthusiasm.

\section{REFERENCES}

[1] Liu Jing-nong, Liu Yun. Undergraduate practical teaching system research and practice of software college in Beijing University of Aeronautics[J]. Compute education, 2007(11):13-17.

[2] Huang Jie, He Zong-jiang. Explore and practice of experiment course based on project[J]. Compute education, 2008(2):30-33

[3] Arthur Tatnall, Gina Reyes. Teaching IT Project Management to PostgraduateBusiness Students: A Practical Approach[J]. Journal of Information Technology Education. 2005(4): 153-166.

[4] L.Mikhailov, P.Tsvetinov. Evaluation of services using a fuzzy analytic hierarchy process[J]. Applied Soft Computing, 2004(5):2333.

[5] He Zhongxiong. Fuzzy Mathematics and It's Applications[M]. TianJin: TianJin Scientific and Technological Publishing House, 1985(Second Edition).

[6] Liu Puyin, Wu Mengda. Fuzzy Theory and It's Applications[M]. BeiJing: National University of Defense Technology Press, 1998. 